\title{
Las Comisiones de Regulación
}

\author{
Hernán Alejandro Olano García* \\ Grupo de Investigación Justicia, Ámbito Público y Derechos Humanos \\ Universidad de La Sabana, Colombia.
}

\section{RESUMEN}

Las Comisiones de Regulación: CREG, CRA, CRC y CRES, han surgido desde 1994, con el propósito de desarrollar la intervención estatal en el ámbito socio-económico para la regulación, de los servicios públicos domiciliarios mediante asignación de la propia ley o en virtud de delegación por parte del Presidente de la República, para promover y garantizar la competencia entre quienes los presten, se sujetarán en cuanto a su estructura, organización y funcionamiento a lo dispuesto en los correspondientes actos de creación, que las considera como unidades administrativas especiales, con independencia administrativa, técnica y patrimonial, y adscritas al ministerio de la materia correspondiente a su objetivo.

\section{PALABRAS CLAVE}

Comisiones de Regulación, Desconcentración Administrativa, CRA, CREG, CRES, CRC, Presidente de la República, Estructura del Estado.

\section{DESARROLLO}

El presente artículo desarrolla un estado del arte sobre la situación actual de las Comisiones de Regulación, dentro de la línea de investigación en Historia de las Instituciones; esa labor de las Comisiones de Regulación corresponde a un tipo de función desconcentrada. La desconcentración también se conoce con el nombre de "descentralización burocrática". Se dice que Es una fórmula intermedia entre centralización y descentralización administrativas. Se caracteriza por el traslado de determinados poderes de administración del órgano central a los inferiores, con el fin de descongestionarlo, facilitando así la resolución de los asuntos en forma más rápida y a veces menos gravosa que si los interesados tuvieran que hacerlo acudiendo al superior jerárquico, sin romper la vinculación jerárquica entre dichos órganos y con la advertencia de que los poderes transferidos son propios del poder central yno de los órganos inferiores.

\section{La Ley 489 de 1998 la define en su artículo $8^{\circ}$ la desconcentración, así:}

*Abogado, con estancia Post Doctoral en Derecho Constitucional como Becario de la Fundación Carolina en la Universidad de Navarra, España; Doctor Magna Cum Laude en Derecho Canónico; es Magíster en Relaciones Internacionales y Magíster en Derecho Canónico y posee especializaciones en Bioética, Derechos Humanos, Liderazgo Estratégico Militar y Derecho Constitucional. Es el Director del Grupo de Investigación en Derecho Público "Diego de Torres y Moyachoque, Cacique de Turmequé", en la Universidad de La Sabana, donde además hace parte del Grupo de Investigación en "Justicia, Ámbito Público y Derechos Humanos" y dirige el Área de Derecho Administrativo y Tributario. Imparte las asignaturas: Derecho Administrativo General y la electiva Derecho Procesal Constitucional en la Universidad de La Sabana. Es además el Vicecónsul Honorario de la República de Chipre en Colombia. Fue Secretario General (e.) de la Corte Constitucional de Colombia, Director General Jurídico y Asesor del Despacho del Viceministro en el Ministerio del Interior; Director Nacional para la prevención del lavado de activos en la Caja Agraria de Colombia y Director de Control Interno en el Concejo de Bogotá. Es Columnista del Diario "El Nuevo Siglo" de Bogotá. Investigador de la Cátedra Garrigues, Miembro de Número de la Red Iberoamericana de Profesores e Investigadores de Derecho Administrativo. Miembro de Número de la Academia Colombiana de Jurisprudencia, Miembro Correspondiente de la Academia Chilena de Ciencias Sociales, Políticas y Morales, y Miembro del Centro Colombiano de Derecho Procesal Constitucional. http://hernanolano.googlepages.com Correo electrónico hernan.olano@unisabana.edu.co Dirección: Universidad de La Sabana, Km. 7 Autopista Norte, Costado Occidental, Chía, Cundinamarca, Colombia. 
"La desconcentración es la radicación de competencias y funciones en dependencias ubicadas fuera de la sede principal del organismo - entidad administrativa, sin perjuicio de las potestades y deberes de onentación e instrucción que corresponde ejercer a los jefes superiores de la Administración, la cual no implica delegación y podrá hacerse por ternitorio y porfunciones.

Parágrafo. En el acto correspondiente se determinarán los medios necesarios para su adecuado cumplimiento.

Los actos cumplidos por las autoridades en virtud de desconcentración administrativa sólo serán susceptibles del recurso de reposición en los términos establecidos en las normas pertinentes."

\section{Características de la Desconcentración:}

- Está dispuesta por la Ley.

- La desconcentración es permanente, y para asumir la competencia o la función se requiere nueva Ley.

- Limitación del ejercicio de la competencia al órgano en quien se desconcentra.

- Se atribuyen competencias limitadas, o funciones a órganos jerárquicamente subordinados.

\section{Clases de Desconcentración:}

* Territorial, horizontal o periférica y central, vertical o simplemente jerárquica.

* Por delegación y por adscripción o por asignación legal.

* Territorial, horizontal o periférica y central, vertical o simplemente jerárquica.

- La desconcentración territorial, se presenta cuando se da el desplazamiento de funciones administrativas del sector central hacia las circunscripciones.

- La desconcentración territorial o periférica, se presenta cuando el ordenamiento jurídico distribuye competencias a dependencias territoriales, porfuera del sector central.

- La desconcentración simplemente jerárquica, es aquella cuando se desplazan funciones administrativas de las autoridades superiores a sus subalternos o subordinados sin que haya desplazamiento territorial.

- La desconcentración central o simplemente jerárquica se da cuando el orden jurídico distribuye competencias dentro del mismo órgano central.

* Por delegación y por adscripción o por asignación legal.

- En el primer caso hay un delegante que es titular de las funciones administrativas y las traslada al delegatario, que es inferior, subalterno o subordinado, para que las ejerza en nombre del delegante. Es una delegación interna o unilateral.

- En el evento de la adscripción o asignación legal, la Ley otorga directamente las funciones administrativas a un funcionario inferior, subalterno o subordinado, que en principio corresponderían a su superior.

Encontramos en éste núcleo el Banco de la República: banco central de Colombia; la Comisión Nacional de Televisión; las comisiones de regulación; los entes de naturaleza única y las entidades privadas con funciones públicas y la función pública en manos de particulares.

En la Sentencia C-041 de 2003, M.P., Dr. Jaime Córdoba Triviño, la Corte Constitucional dispuso que:

“... los servicios públicos se caracterizan por: (i) tener una connotación eminentemente social, en tanto que pretenden el bienestar y mejoramiento de la calidad de vida de las personas y por ello su prestación debe ser eficiente; (ii) el régimen jurídico al cual estarán sometidos es el que fije la ley; (iii) pueden ser prestados no solamente por el Estado, directa o indirectamente, sino también por comunidades organizadas o por particulares; (iv) el Estado mantendrá siempre su regulación, control y vigilancia; (v) su régimen tarifario consultará, además de los criterios de costos, los de solidaridad y redistribución de ingresos; (vi) deberán ser prestados directamente por los municipios, en tratándose de los servicios públicos 
domicilianios, cuando las características técnicas y económicas del servicio y las conveniencias generales 10 permitan y aconsejen, y (vii) las entidades territoriales pueden conceder subsidios para las personas de menores ingresos."

En la Sentencia C-150 de 2003, no obstante la ineptitud de la demanda de inconstitucionalidad formulada en esa oportunidad, la Corte Constitucional se refirió a la función estatal de regulación, en los siguientes términos:

"En un Estado social de derecho la intervención estatal en el ámbito socio-económico puede obedecer al cumplimiento de diversas funciones generalmente agrupadas en cuatro grandes categorías: una función de redistribución del ingreso $y$ de la propiedad expresamente consagrada en varias disposiciones de la Constitución con miras a alcanzar un "orden politico, económico y social justo" (Preámbulo); una función de estabilización económica también consagrada en diversas normas superiores (artículos 334 inc, $1^{\circ}, 339,347$, 371 y 373 de la C.P.); una función de regulación económica y social de múltiples sectores y actividades especificas según los diversos parámetros trazados en la Constitución (artículos 49 y 150, numeral 19, por ejemplo); y, todas las anteriores, dentro de un contexto de intervención general encaminado a definir las condiciones fundamentales del funcionamiento del mercado y de la convivencia social, como el derecho de propiedad privada pero entendido como "función social" (artículo 58 C.P.) o la libertad de iniciativa privada y de la actividad económica siempre que se respete también la "función social" de la empresa (artículo 333 C.P.) en aras de la "distribución equitativa de las oportunidades y los beneficios del desarrollo" (artículo 334 C.P.).

\section{(...)}

No obstante, en determinadas circunstancias históricas, esta forma básica de regulación socio-económica ha sido considerada inadecuada para alcanzar ciertos objetivos sociales valorados por una comunidad politica, como, por ejemplo, la disminución de la pobreza y de las desigualdades sociales o la protección de un ambiente sano. Además, la dinámica misma del mercado, asi regulado, puede llevar a consecuencias indeseables en el plano macroeconómico, como concentración de la propiedad en pocas manos, altas tasas de desempleo, ciclos económicos profundos y prolongados, entre otras. Por ello, desde hace un siglo, el Estado ha intervenido con fines de protección social, de redistribución o de estabilización económica.

\section{(...)}

A estos elementos de la función estatal de regulación, se puede sumar otro que ha conducido a que el esquema de regulación adoptado por el constituyente 0 el legislador adquiera rasgos específicos. En efecto, en algunos sectores, se presenta la necesidad de proteger los derechos de las personas. Cuando ello ocurre, la función de regulación se orienta en sus aspectos estructurales, instrumentales y procedimentales al cumplimiento de esa finalidad primordial. Es lo que sucede en el sector de los servicios públicos donde la Constitución ha protegido específicamente los derechos de los usuarios (artículos 78 y 369 C.P.). Ello conduce a que en estos ámbitos la función de regulación estatal esté orientada constitucionalmente al logro de unos fines sociales también específicos como los de redistribución y solidaridad en el ámbito de los servicios públicos domiciliarios (artículo 367 C.P.) 0 el de acceso universal en todos los servicios (artículo 365 C.P.)."

Según Gordillo', "debemos empezar a olvidar los entes administrativos descentralizados, sometidos al control de tutela de la administración central y comenzar a enfatizar que el Estado contemporáneo requiere de autoridades administrativas independientes del poder central", los cuales se ocupan en sede administrativa de la protección de los derechos e intereses de los usuarios. 
El artículo 68 de la Ley 142 de 1994 creó las Comisiones de Regulación, en los siguientes términos:

\section{DE IAS COMISIONES DE REGULACIÓN}

ART. 68. Delegación de funciones presidenciales a las Comisiones. El Presidente de la República señalará las políticas generales de administración y control de eficiencia de los servicios públicos domiciliarios, que le encomienda el artículo 370 de la Constitución Política, y de los demás a los que se refiere esta Ley, por medio de las Comisiones de Regulación de los servicios públicos, si decide delegarlas, en los términos de esta Ley.

Las normas de esta Ley que se refieren a las Comisiones de Regulación se aplicarán si el Presidente resuelve delegar la función aludida; en caso contrario, el Presidente ejercerá las funciones que aqui se atribuyen a las Comisiones.

Y, el Estatuto de Organización y Funcionamiento de la Administración pública, Ley 489 de 1998, en su artículo 48 indica lo siguiente:

\section{ARTICULO 48. COMISIONES DE REGULACION.} Las comisiones que cree la ley para la regulación, de los servicios públicos domiciliarios mediante asignación de la propia ley o en virtud de delegación por parte del Presidente de la República, para promovery garantizarla competencia entre quienes los presten, se sujetarán en cuanto a su estructura, organización y funcionamiento a lo dispuesto en los correspondientes actos de creación.

Creadas como unidades administrativas especiales, con independencia administrativa, técnica y patrimonial, y adscritas al respectivo ministerio, tenemos, según el artículo 69 de la Ley 142 de 1994 las siguientes Comisiones de Regulación:

- Comisión de Regulación de Energía y Gas CREG, adscrita al Ministerio de Minas y Energía.
- Comisión de Regulación de las Comunicaciones - CRC, antes CRT, adscrita al Ministerio de las Nuevas Tecnologías de la Información y las Comunicaciones.

- Comisión de Regulación de Agua Potable y Saneamiento Básico - CRA, adscrita al Ministerio de Medio Ambiente, Vivienda y Desarrollo Territorial.

- Comisión de Regulación en Salud - CRES, creada por la Ley 1122 de 2006.

Cada comisión será competente para regular el servicio público respectivo.

Las Comisiones de Regulación tienen la función de regular los monopolios en la prestación de los servicios públicos, cuando la competencia no sea, de hecho, posible; y, en los demás casos, la de promover la competencia entre quienes presten servicios públicos, para que las operaciones de los monopolistas o de los competidores sean económicamente eficientes, no impliquen abuso de la posición dominante, y produzcan servicios de calidad. Para ello tendrán las siguientes funciones y facultades especiales, de acuerdo con el artículo 73 de la Ley 142 de 1994:

- Preparar proyectos de Ley para someter a la consideración del gobierno, y recomendarle la adopción de los decretos reglamentarios que se necesiten.

- Someter a su regulación, a la vigilancia del Superintendente, y a las normas que esta Ley contiene en materia de tarifas, de información y de actos y contratos, a empresas determinadas que no sean de servicios públicos, pero respecto de las cuales existan pruebas de que han realizado o se preparan para realizar una de las siguientes conductas:

a. Competir deslealmente con las de servicios públicos;

b. Reducir la competencia entre empresas de servicios públicos;

c. Abusar de una posición dominante en la provisión de bienes o servicios similares a los que éstas ofrecen. 
- Definir los criterios de eficiencia y desarrollar indicadores y modelos para evaluar la gestión financiera, técnica y administrativa de las empresas de servicios públicos y solicitar las evaluaciones que considere necesarias para el ejercicio de sus funciones.

- Fijar las normas de calidad a las que deben cen̂irse las empresas de servicios públicos en la prestación del servicio.

- Definir en qué eventos es necesario que la realización de obras, instalación y operación de equipos de las empresas de servicios públicos se someta a normas técnicas oficiales, para promover la competencia o evitar perjuicios a terceros, y pedirle al ministerio respectivo que las elabore, cuando encuentre que son necesarias.

- Establecer la cuantia y condiciones de las garantías de seriedad que deben prestar quienes deseen celebrar contratos de aporte reembolsable.

- Decidir los recursos que se interpongan contra sus actos, o los de otras entidades, en los casos que disponga la Ley en lo que se refiere a materias de su competencia.

- Resolver, a petición de cualquiera de las partes, los conflictos que surjan entre empresas, por razón de los contratos o servidumbres que existan entre ellas y que no corresponda decidir a otras autoridades administrativas. La resolución que se adopte estará sujeta al control jurisdiccional de legalidad.

- Resolver, a petición de cualquiera de las partes, los conflictos que surjan entre empresas, y que no corresponda decidir a otras autoridades administrativas, acerca de quién debe servir a usuarios específicos, o en qué regiones deben prestar sus servicios. La resolución que se adopte estará sujeta al control jurisdiccional de legalidad. La resolución debe atender, especialmente, al propósito de minimizar los costos en la provisión del servicio.

- Dar concepto sobre la legalidad de las condiciones uniformes de los contratos de servicios públicos que se sometan a su consideración; y sobre aquellas modificaciones que puedan considerarse restrictivas de la competencia. Las Comisiones podrán limitar, por vía general, la duración de los contratos que celebren las empresas de servicios públicos, para evitar que se limite la posibilidad de competencia.

- Establecer fórmulas para la fijación de las tarifas de los servicios públicos, cuando ello corresponda según lo previsto en el artículo 88 ; y señalar cuándo hay suficiente competencia como para que la fijación de las tarifas sea libre.

- Determinar para cada bien o servicio público las unidades de medida y de tiempo que deben utilizarse al definir el consumo; y definir, con bases estadísticas y de acuerdo con parámetros técnicos medibles y venificables, apropiados para cada servicio, quiénes pueden considerarse "grandes usuarios".

- Ordenar que una empresa de servicios públicos se escinda en otras que tengan el mismo objeto de la que se escinde, o cuyo objeto se limite a una actividad complementaria, cuando se encuentre que la empresa que debe escindirse usa su posición dominante para impedir el desarrollo de la competencia en un mercado donde ella es posible; o que la empresa que debe escindirse otorga subsidios con el producto de uno de sus servicios que no tiene amplia competencia a otro servicio que sí la tiene; 0 , en general, que adopta prácticas restrictivas de la competencia.

- Ordenar la fusión de empresas cuando haya estudios que demuestren que ello es indispensable para extender la cobertura y abaratar los costos para los usuarios.

- Ordenar la liquidación de empresas monopolísticas oficiales en el campo de los servicios públicos y otorgar a terceros el desarrollo de su actividad, cuando no cumplan los requisitos de eficiencia a los que se refiere la Ley 142.

- Impedir que quienes captan o producen un bien que se distribuye por medio de empresas de servicios públicos adopten pactos contrarios a la libre competencia en perjuicio de los distribuidores; y exigir que en los contratos se especifiquen los diversos componentes que definen los precios y tarifas.

- Dictar los estatutos de la comisión y su propio reglamento, y someterlos a aprobación del Gobierno Nacional. 
- Pedir al Superintendente que adelante las investigaciones e imponga las sanciones de su competencia, cuando tenga indicios de que alguna persona ha violado las normas de la Ley de Servicios Públicos.

- Resolver consultas sobre el régimen de incompatibilidades e inhabilidades al que se refiere la Ley de Servicios Públicos.

- Determinar, de acuerdo con la Ley, cuándo se establece el régimen de libertad regulada 0 libertad vigilada o señalar cuándo hay lugar a la libre fijación de tarifas.

- Señalar, de acuerdo con la Ley, criterios generales sobre abuso de posición dominante en los contratos de servicios públicos, y sobre la protección de los derechos de los usuarios en lo relativo a facturación, comercialización y demás asuntos relativos a la relación de la empresa con el usuario.

- Establecer los requisitos generales a los que deben someterse las empresas de servicios públicos para utilizar las redes existentes $y$ acceder a las redes públicas de interconexión; así mismo, establecer las fórmulas tarifarias para cobrar por el transporte e interconexión a las redes, de acuerdo con las reglas de la Ley de Servicios Públicos.

- Definir cuáles son, dentro de las tarifas existentes al entrar en vigencia la Ley 142, los factores que se están aplicando para dar subsidios a los usuarios de los estratos inferiores, con el propósito de que esos mismos factores se destinen a financiar los fondos de solidaridad y redistribución de ingresos, y cumplir así lo dispuesto en el numeral 87.3 de la Ley 142.

- Absolver consultas sobre las materias de su competencia.

- Establecer los mecanismos indispensables para evitar concentración de la propiedad accionaria en empresas con actividades complementarias en un mismo sector o sectores afines en la prestación de cada servicio público.

- Todas las demás que le asigne la Ley y las facultades previstas en ella que no se hayan atribuido a una autoridad específica.
Salvo cuando la Ley de Servicios Públicos diga lo contrario en forma explícita, no se requiere autorización previa de las Comisiones para adelantar ninguna actividad o contrato relacionado con los servicios públicos; ni el envío rutinario de información. Pero las Comisiones, tendrán facultad selectiva de pedir información amplia, exacta, veraz y oportuna a quienes prestan los servicios públicos a los que la Ley 142 se refiere, inclusive si sus tarifas no están sometidas a regulación. Quienes no la proporcionen, estarán sujetos a todas las sanciones que contempla el artículo 81 de la presente Ley. En todo caso, las Comisiones podrán imponer por sí mismas las sanciones del caso, cuando no se atiendan en forma adecuada sus solicitudes de información.

Para el cumplimiento de las funciones que les asigna la Ley 142, en el evento de la delegación presidencial, las comisiones de regulación tendrán la siguiente estructura orgánica, que el Presidente de la República modificará, cuando sea preciso, previo concepto de la comisión respectiva dentro de las reglas del artículo 105 de la Ley 142.

Comisión de Regulación:

1. Comité de Expertos Comisionados

2. Coordinación General:

3. Coordinación Ejecutiva

4. Coordinación Administrativa

5. Áreas Ejecutoras

6. Oficina de regulación y políticas de competencia

7. Oficina Técnica

8. Oficina Jurídica

De acuerdo con el artículo 71 de la citada Ley 142 de 1994 y la Ley 373 de 1997, las Comisiones de Regulación estarán integradas por:

- El Ministro respectivo o su delegado, quien la presidirá.

- A la Comisión de Regulación de Agua Potable y Saneamiento Básico pertenecerán los Ministros de Protección Social y Ambiente, Vivienda y Desarrollo Territorial. A la Comisión de Regulación de Energía y Gas Combustible pertenecerá el Ministro de Hacienda y Crédito Público. Los ministros sólo podrán delegar su 
asistencia en los viceministros y el director del Departamento Nacional de Planeación en el Subdirector. ${ }^{2}$

- El Director del Departamento Nacional de Planeación.

- A las comisiones asistirá, únicamente con voz, el Superintendente de Servicios Públicos o su delegado. Así mismo, cada una posee ciertas particularidades en cuanto a su composición.

La Comisión de Regulación de Energía y Gas Combustible, CREG.

Deacuerdo con la Ley 142 de 1994, se encarga de:

a. Regular el ejercicio de las actividades de los sectores de energía y gas combustible para asegurar la disponibilidad de una oferta energética eficiente, propiciar la competencia en el sector de minas y energía y proponer la adopción de las medidas necesarias para impedir abusos de posición dominante y buscar la liberación gradual de los mercados hacia la libre competencia. La Comisión podrá adoptar reglas de comportamiento diferencial, según la posición de las empresas en el mercado;

b. Expedir regulaciones específicas para la autogeneración y cogeneración de electricidad y el uso eficiente de energía y gas combustible por parte de los consumidores y establecer criterios para la fijación de compromisos de ventas garantizadas de energía y potencia entre las empresas eléctricas y entre éstas y los grandes usuarios;

c. Establecer el reglamento de operación para realizar el planeamiento y la coordinación de la operación del sistema interconectado nacional y para regular el funcionamiento del mercado mayorista de energía y gas combustible.

d. Fijar las tarifas de venta de electricidad y gas combustible; 0 delegar en las empresas distribuidoras, cuando sea conveniente dentro de los propósitos de esta Ley, bajo el régimen que ella disponga, la facultad de fijar estas tarifas. e. Definir las metodologías y regular las tarifas por los servicios de despacho y coordinación prestados por los centros regionales y por el centro nacional de despacho.

\section{La Comisión de Regulación de Agua Potable y Saneamiento Básico-CRA:}

Según la Ley 373 de 1997, la Comisión de Regulación de Agua Potable y Saneamiento Básico estará integrada por: Cuatro expertos comisionados de dedicación exclusiva, designados por el Presidente de la República para período de 3 años, reelegibles y no sujetos a las disposiciones que regulan la carrera administrativa. Uno de ellos en forma rotatoria ejercerá las funciones de coordinador de acuerdo con el reglamento interno. Al repartir internamente el trabajo entre ellos se procurará que todos tengan oportunidad de prestar sus servicios respecto de las diversas clases de asuntos que son competencia de la Comisión. En todo caso, uno de los expertos deberá demostrar conocimientos en materias ambientales.

Sus funciones, con base en la Ley 142 de 1994 , son las siguientes:

a. Promover la competencia entre quienes presten los servicios de agua potable y saneamiento básico o regular los monopolios en la prestación de tales servicios, cuando la competencia no sea posible, todo ello con el propósito de que las operaciones de los monopolistas y de los competidores sean económicamente eficientes, se prevenga el abuso de posiciones dominantes y se produzcan servicios de calidad. La Comisión podrá adoptar reglas de comportamiento diferencial, según la posición de las empresas en el mercado;

b. Establecer, por vía general, en qué eventos es necesario que la realización de obras, instalaciones y operación de equipos destinados a la prestación de servicios de acueducto, alcantarillado y aseo se sometan a normas técnicas y adoptar las medidas necesarias para que se apliquen las normas técnicas sobre calidad de agua potable que establezca el Ministerio de Salud, en tal forma que 
se fortalezcan los mecanismos de control de calidad de agua potable por parte de las entidades competentes.

\section{La Comisión Reguladora de Comunicaciones, CRC, antes CRT:}

Su denominación fue modificada en el año 2009, cuando se cambió el nombre del Ministerio de Comunicaciones por el de Nuevas Tecnologías de la Información y las Comunicaciones. (Ley 1341 de 2009). Según la Ley 142 de 1994, debe cumplir las siguientes funciones específicas:

a. Promover la competencia en el sector de las telecomunicaciones, y proponer 0 adoptar las medidas necesarias para impedir abusos de posición dominante, pudiendo proponer reglas de comportamiento diferenciales según la posición de las empresas en el mercado.

b. Resolver los conflictos que se presenten entre operadores en aquellos casos en los que se requiera la intervención de las autoridades para garantizar los principios de libre y leal competencia en el sectory de eficiencia en el servicio.

c. Establecer los requisitos generales a que deben someterse los operadores de servicios de telefonía básica de larga distancia nacional e internacional para ejercer el derecho a utilizar las redes de telecomunicaciones del estado; así mismo, fijar los cargos de acceso y de interconexión a estas redes, de acuerdo con las reglas sobre tarifas previstas en esta Ley.

d. Reglamentar la concesión de licencias para el establecimiento de operadores de servicios de telefonía básica de larga distancia nacional e internacional, y señalar las fórmulas de tarifas que se cobrarán por la concesión.

e. Definir, de acuerdo con el tráfico cursado, el factor de las tarifas de servicios de telefonía básica de larga distancia nacional e internacional, actualmente vigentes, que no corresponde al valor de la prestación del servicio. Parte del producto de ese factor, en los recaudos que se hagan, se asignará en el Presupuesto Nacional, previo concepto del Consejo Nacional de Política Económica y Social, para el "Fondo de Comunicaciones del Ministerio", que tendrá a su cargo hacer inversión por medio del fomento de programas de telefonía social, dirigidos a las zonas rurales y urbanas caracterizadas por la existencia de usuarios con altos índices de necesidades básicas insatisfechas. Se aplicarán a este fondo, en lo pertinente, las demás normas sobre "fondos de solidaridad y redistribución de ingresos" a los que se refiere el artículo 89 de esta Ley. En el servicio de larga distancia internacional no se aplicará el factor de que trata el artículo 89 y los subsidios que se otorguen serán financiados con recursos de ingresos ordinarios de la nación y las entidades territoriales.

f. Proponer al mismo consejo la distribución de los ingresos de las tarifas de concesiones de servicio de telefonia móvil celular y de servicios de larga distancia nacional e internacional, para que este determine en el proyecto de presupuesto qué parte se asignará al fondo atrás mencionado y qué parte ingresará como recursos ordinarios de la nación y definir el alcance de los programas de telefonía social que elabore el Fondo de Comunicaciones.

\section{Comisión de Regulación en Salud-CRES:}

La Ley 1122 de 2006, dispuso la creación de la Comisión de Regulación en Salud (CRES), como unidad administrativa especial, con personeria jurídica, autonomía administrativa, técnica y patrimonial, adscrita al Ministerio de la Protección Social.Con base en esa normatividad, el Consejo Nacional de Seguridad Social en Salud, con carácter de asesor y consultor del Ministerio de la Protección Social y de la Comisión de Regulación en Salud, mantendrá vigentes sus funciones establecidas en la Ley 100 de 1993, mientras no entre en funcionamiento la Comisión de Regulación en Salud CRES.

El Ministerio de la Protección Social reglamentará las funciones de asesoría y consultoría del Consejo Nacional de Seguridad Social en Salud.

La Corte Constitucional encontró que si bien en la exposición de motivos y en las ponencias de trámite 
no se hizo una análisis de impacto fiscal concretamente dirigido a estudiar los costos que generaría la creación de la CRES, las fuentes de recursos para atender ese nuevo costo y la compatibilidad de dicho gasto con el Marco Fiscal de Mediano Plazo, lo cierto es que, en dichas exposición y ponencia sí se hizo un estudio de esta naturaleza que englobaba la financiación general de la reforma al Sistema de Seguridad Social en Salud y las fuentes de nuevos recursos que engrosarían el FOSYGA, recursos éstos últimos llamados a cubrir los costos de la nueva entidad de regulación. Además, como se puede apreciar en las actas respectivas, en el curso de los debates se hizo referencia y se reflexionó acerca de de los costos fiscales que crearía la creación de la CRES, de manera que fue un asunto considerado y sopesado por el Congreso, que para el efecto tuvo la oportunidad de consultar la opinión del Viceministro de Hacienda y el Ministro de la Protección Social, quienes emitieron un concepto favorable a este respecto. La norma fue declarada Exequible por medio de la Sentencia C 955 de 2007. Corte Constitucional. Comunicado de Prensa 49 de 2007.

La Comisión de Regulación en Salud estará integrada de la siguiente manera:

1. El Ministro de la Protección Social quien la preside, excepcionalmente podrá delegar sólo en alguno de sus Viceministros.

2. El Ministro de Hacienda y Crédito Público quien, excepcionalmente podrá delegar sólo en alguno de sus Viceministros.

3. Cinco Comisionados expertos, designados por el Presidente de la República, de temas enviadas por diferentes entidades tales como: Asociación Colombiana de Universidades, Centros de Investigación en Salud, Centros de Investigación en Economia de la Salud, Asociaciones de Profesionales de la Salud y Asociaciones de Usuarios debidamente organizados. El Gobierno Nacional reglamentará la materia.

Las temas serán elaboradas por las anteriores organizaciones, a partir de una lista de elegibles conformada mediante concurso público de méritos para todas las profesiones que incluyan examen de antecedentes laborales, examen de conocimientos sobre el Sistema General de Seguridad Social en Salud, de acuerdo con su experiencia y entrevista conforme lo señale el reglamento.

Los comisionados expertos de la Comisión de Regulación en Salud serán de dedicación exclusiva.

Los anteriores comisionados ejercerán por períodos individuales de tres (3) años, reelegibles por una sola vez y no estarán sujetos a las disposiciones que regulan la carrera administrativa.

Los comisionados estarán sujetos al siguiente régimen de inhabilidades e incompatibilidades:

Los comisionados no podrán tener directa o a través de terceros ningún vínculo contractual 0 comercial con entidades promotoras de salud, instituciones prestadoras de servicios de salud y productoras o comercializadores mayoristas de medicamentos o insumos hospitalarios.

No podrán ser comisionados aquellas personas cuyo cónyuge o compañero (a) permanente, o sus parientes en el tercer grado de consaguinidad, segundo de afinidad o primero civil, sean representantes legales, miembros de junta directiva 0 accionistas 0 propietarios de entidades promotoras de salud, instituciones prestadoras de servicios de salud, empresas productoras o comercializadoras mayoristas de medicamentos 0 insumos hospitalarios.

Los expertos deberán ser profesionales mínimo con título de maestría o su equivalente, cada uno de ellos deberá acreditar experiencia en su respectiva área no menor de 10 años.

La Comisión de Regulación en Salud ejercerá las siguientes funciones:

a. Definir y modificar los Planes Obligatorios de Salud (POS) que las Entidades Promotoras de Salud (EPS) garantizarán a los afiliados 
según las normas de las Regímenes Contributivo y Subsidiado.

b. Definir y revisar, como mínimo una vez al año, el listado de medicamentos esenciales y genéricos que harán parte de los Planes de Beneficios.

c. Definir el valor de la Unidad de Pago por Capitación de cada Régimen, de acuerdo con la presente Ley. Si a 31 de diciembre de cada año, la Comisión no ha aprobado un incremento en el valor de la UPC, dicho valor se incrementará automáticamente en la inflación causada.

d. Definir el valor por beneficiaria de los subsidios parciales en salud, sus beneficios y las mecanismos para hacer efectivo el subsidio.

e. Definir los criterios para establecer los pagos moderadores de que trata el numeral 30 del artículo 160 y los artículos 164 y 187 de la Ley 100 de 1993. f. Definir el régimen que deberán aplicar las EPS para el reconocimiento y pago de las incapacidades originadas en enfermedad general $o$ en las licencias de maternidad, según las normas del Régimen Contributivo.

g. (Establecer y actualizar un Sistema de tarifas que debe contener entre otros componentes, un manual de tarifas mínimas que será revisado cada año, incluyendo los honorarios profesionales. En caso de no revisarse el misma, será indexado con la inflación causada).

h. Presentar ante las Comisiones Séptimas de Senado y Cámara, un informe anual sobre la evolución del Sistema General de Seguridad Social en Salud y las recomendaciones para mejorarla.

i. Recomendar proyectos de ley 0 de decretos reglamentarios cuando a su juicio sean requeridos en el ámbito de la salud.

j. Adoptar su propio reglamento.

\section{CONCLUSIONES}

La intervención estatal obedece a las funciones de redistribución del ingreso y de la propiedad expresamente consagrada en varias disposiciones de la Constitución con miras a alcanzar un "orden político, económico y social justo" (Preámbulo); una función de estabilización económica también consagrada en diversas normas superiores (artículos 334 inc, $1^{\circ}, 339,347,371$ y 373 de la C.P.); una función de regulación económica y social de múltiples sectores y actividades específicas según los diversos parámetros trazados en la Constitución (artículos 49 y 150, numeral 19, por ejemplo); y, todas las anteriores, se cumplen dentro del contexto de la desconcentración administrativa, ese término medio entre la centralización y la descentralización, que lo único que permite es agigantar el tamaño del Estado y con esas moléculas independientes, evitar que el átomo, el Estado, cumpla su verdadera función: La búsqueda del bien común. 